\title{
Life Cycle Cost Estimation - Core Requirements
}

\author{
Real Jagdishsingh ${ }^{1}$, Rahul S Patil ${ }^{2}$ \\ P.G student Department of Civil Engineering, Padamshree Dr. D. Y Patil institute of Engineering \& \\ Technology. Pimpri Pune. \\ Assistant Professor in Department of Civil Engineering, Padamshree Dr. D. Y Patil institute of Engineering \& \\ Technology. Pimpri Pune.
}

\begin{abstract}
There are many possible sources of data that can be used in cost estimates for life Cycle Costing. Irrespective of the source, the accuracy of the data always remains the responsibility of the cost Estimator. In almost all the cases, the data will need to be adjusted or normalized. For example, the reference system cost should be adjusted to account for any differences in system parameters (it may be technical, physical, complexity, or hardware cost) or operating conditions between the reference system and the proposed system being calculated. The Success of the Life Cycle costing depends on the accuracy of the cost factors and as such huge efforts are needed to map a system which would help in the accurate prediction of the various cost categories.Actual cost incurred on past and current project procurement programs often forms the basis of estimates of future projects. Project managers use the Quantity Survey system to report data on contractor development, production, and sustainment costs and resource usage incurred in construction projects and their facility management. It is important to assess the accuracy of the outputs from the estimating methods and models, drawing conclusions about reasonableness and validity. Peer review is often helpful at this point. For complex cost estimates, with many elements provided from different sources, considerable effort and care are needed to deconflict and synthesize the various elements. This paper explains the importance and process for estimation for $L C C$.
\end{abstract}

Key Words: Life cycle costing, sensitivity analysis, pertmaster, monte carlo simulation.

\section{Introduction}

Life Cycle costing is because of the need to select the best project execution option and the same is not be based on only the initial project construction cost but the cost of operation as well as the maintenance cost also. For any system, estimates of future life-cycle costs are subject to varying degrees of Clarity. The overall ambivalence is not only due to incertitude in cost estimating methods, but also due to ambivalence in program or system definition or in technical performance. Although these uncertainties cannot be eliminated, it is useful to identify associated risk issues and to attempt to quantify the degree of uncertainty as much as possible. This bounding of the cost estimate may be attempted through sensitivity analyses or through a formal quantitative risk analysis. (Risk analysis through programs like primavera pertmaster, etc)

\section{Cost Estimating}

In order to achieve high level of accuracy in LCC sensitivity analysis is required to be performed for the cost estimates, Sensitivity analysis attempts to demonstrate how estimates cost would change if one or more assumptions change. Typically, for the high-cost elements, the estimator identifies the relevant cost-categories, and then determines how costs vary with changes in the cost-category units. For example, a sensitivity analysis might examine how maintenance manning varies with different assumptions about system reliability and maintainability values, or how system manufacturing labor and material costs vary with system weight growth. In good sensitivity analyses, the cost-drivers are not changed by unbiased percentages, but rather by a careful calculation of the underlying risks. Another major source of alternatives can be from the vendors with different systems proposed by them. Sensitivity analysis is useful for identifying critical estimating assumptions In contrast, quantitative risk analysis can provide a broad overall assessment of variability in the cost estimate. In risk analysis, selected factors (technical, programmatic and cost) are described by probability distributions. Where estimates are based on cost models derived from historical data, the effects of cost estimation error may be included in the range of considerations included in the cost risk assessment. Risk analysis assesses the aggregate variability in the overall estimate due to the variability in each input probability distribution, typically through Monte-Carlo simulations. It is then possible to derive an estimated empirical probability distribution for the overall life-cycle cost estimate. This allows the analyst to describe the nature and degree of variability in the estimate.

Sensitivity and risk analyses also have uses beyond addressing the uncertainty in cost estimates. They also can be used to help better understand what can go wrong with a program, and focus appropriate management attention to risk areas that are concerns. The history of past procurement and acquisition would 
indicate that cost growth and schedule delays can occur as a direct result of one or more of the following concerns:

- $\quad$ Lack of design details at the start of development

- Inadequate understanding of design challenges at the start of construction

- $\quad$ Requirements clarity

- $\quad$ instability, or creep

- $\quad$ Failure to acknowledge fund shortages.

- $\quad$ Funding issues in the programming, budgeting or appropriations process

- $\quad$ Failure to identify (or deal with) unrealistic construction cost proposals in competitive source selections (from either the prime or major subcontractors)

- $\quad$ Excessive relationship between development and procurement schedules

- Inadequate understanding of system size and integration challenges

- Failure to achieve design stability by the time of the critical design review

- $\quad$ Failure to achieve stable construction schedule processes by the time of early production

Top 16 dominant building materials in respect of LCI in the database:

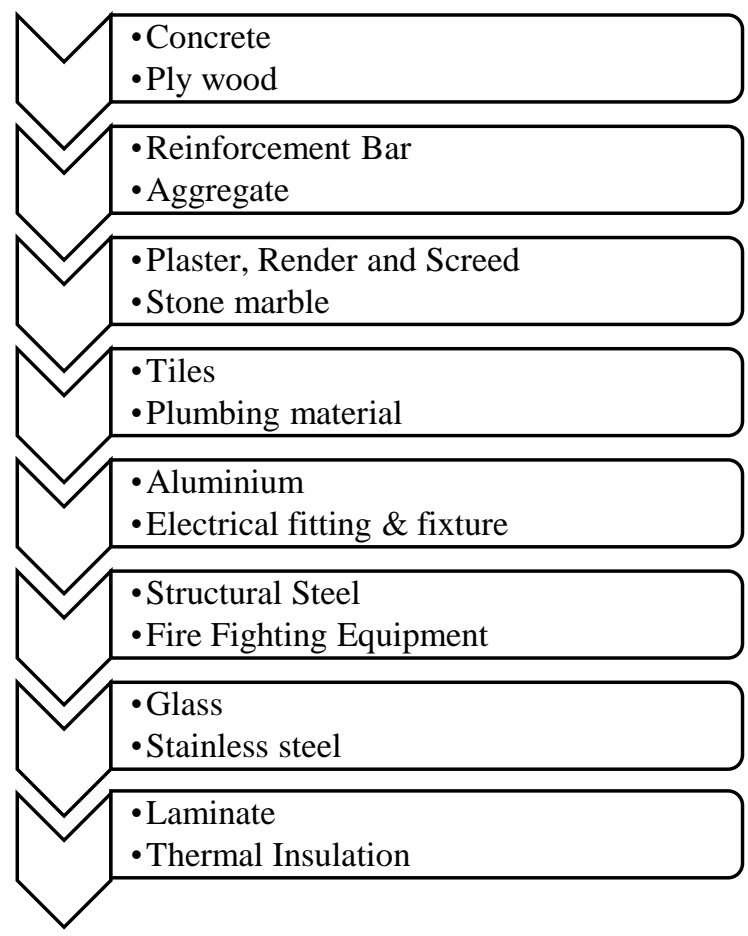

List top 12building services systems \& components with greatest LCI in the database:

$\cdot$ Power cable
$\cdot$ Bus duct




\section{Coordination}

Managing the preparation of a life-cycle cost estimate requires continual coordination among all of the stakeholders. Normally, cost estimates are done by Estimating and costing department and are prepared by a multi-disciplinary team with functional skills in financial management, contracts, engineering, and other talents. The team also should include stakeholders or reviewers from all major involved organizations,. Typically, the analytic approach to the cost estimate is documented in a written study plan that includes a master schedule (of specific tasks, responsible parties, and due dates). For highly complex projects, the estimating team may be organized as a formal Project Estimating Team. Throughout the preparation of the estimate, coordination with all interested parties remains important. Frequent in-progress reviews or meetings are usually a good practice.

For independent cost estimates, the team may be smaller and less formal, but the basic principle-complete and continual coordination of the cost estimate with all interested parties-still applies.

\section{Conclusion}

A complete cost estimate should be formally documented. The documentation serves as an audit trail of source data, methods, and results. The documentation should be easy to read, complete and well organized-to allow any reviewer to understand the estimate fully. The documentation also serves as a valuable reference for future cost analysts, as the program moves from one acquisition milestone to the next.

The documentation should address all aspects of the cost estimate: all ground rules and assumptions; the description of the system and its operating and support concepts; the selection of cost estimating methods; data sources; the actual estimate computations; and the results of any sensitivity or risk analyses. The documentation for the ground rules and assumptions, and the system description, should be written as an updated (final) version of the Cost Analysis Requirements.

Dr. R K Jain. -Principal, DYPIET. Pune.

Acknowledgements

Mr Yashhwant Anantraj. MD SYconepmc. Bangalore.

Mr Charul Vakta. Exe Director YMCAIC. Ahmedabad.

Mr Kiran patel, Engineer project, Sycone CPMC Pvt. Ltd. Ahmedabad,

\section{References}

[1]. Cost and software data (reporting) CSDR system

[2]. ISO 15686-5: Building and construction assets-service life planning (draft)

[3]. NS 3453: Life cycle cost for building and civil engineering work- principles and classification

[4]. Task Group 4: Life cycle cost in construction

[5]. Procurement guide 07: Whole - life costing and cost management

[6]. The Green book by HM Treasury, UK

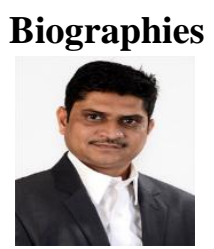

Real Jagdish is a Post Graduate student at Padamshree Dr. D.Y Patil Institute of Engineering and Technology Pimpri, Pune 400018 and Senior Manager Projects With the reputed PMC Firm Sycone Cpmc. Bangalore. He is credited with successful completion of projects like YMCA International Centre at Ahmedabad.

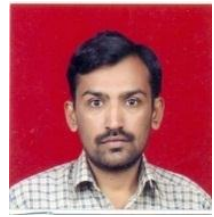

Rahul Patil is Assistant Professor in department of Civil Engineering, Padamshree Dr. D.Y Patil Institute of Engineering and Technology Pimpri, Pune 400018. With more than 12 years' experience in teaching and currently pursuing Ph.D. from University of Pune 